\title{
Divergent Kinetic Control of Classical vs. Ozonolytic Lactonization: Mechanism-based Diastereoselection
}

\author{
Thomas R. Hoye* and Troy D. Ryba \\ 207 Pleasant Street, SE, Department of Chemistry, \\ University of Minnesota, Minneapolis, MN 55455 \\ hoye@chem.umn.edu
}

\section{Supporting Information}

Spectroscopic characterization data for all new compounds S-1 to S-11 Typical NMR spectra used to deduce product ratios S-12 to S-15

\section{General Information.}

${ }^{1} \mathrm{H}$ and ${ }^{13} \mathrm{C}$ NMR spectra were recorded on a Varian VI-500, VI-300, or VXR-300 spectrometer. Proton chemical shifts are referenced to TMS $(0.00 \mathrm{ppm})$ for spectra obtained in $\mathrm{CDCl}_{3}$. Non-first order multiplets are identified as "nfom." Carbon chemical shifts are referenced to $\mathrm{CDCl}_{3}(77.23 \mathrm{ppm})$. High resolution mass spectra were recorded on a Bruker Biotof II instrument. The nomenclature, while systematic and uniform, does not conform fully to either CAS or IUPAC usage. Rather, peloruside A numbering is used throughout to maintain consistency among structures and for ease in tracking assignments of the NMR data. 
$(2 S, 3 R, 7 R, 8 S)$-Dimethyl 5-Hydroxy-3,7-dimethoxy-2,8-bis(methoxymethoxy)nonanedioate (1)

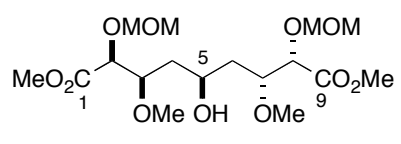

1

${ }^{1} \mathbf{H}$ NMR $\left(500 \mathrm{MHz}, \mathrm{CDCl}_{3}\right): \delta=4.73\left(\mathrm{~d}, J=6.8 \mathrm{~Hz}, 1 \mathrm{H}, \mathrm{OCH}_{\mathrm{al}} \mathrm{HOMe}\right), 4.72(\mathrm{~d}, J=6.8 \mathrm{~Hz}, 1 \mathrm{H}$, $\left.\mathrm{OCH}_{\mathrm{a} 2} \mathrm{HOMe}\right), 4.71\left(\mathrm{~d}, J=6.8 \mathrm{~Hz}, 1 \mathrm{H}, \mathrm{OCH} H_{\mathrm{b} 1} \mathrm{OMe}\right), 4.70\left(\mathrm{~d}, J=6.8 \mathrm{~Hz}, 1 \mathrm{H}, \mathrm{OCH} H_{\mathrm{b} 2} \mathrm{OMe}\right), 4.27(\mathrm{~d}, J$ $=4.2 \mathrm{~Hz}, 1 \mathrm{H}, \mathrm{H}-2$ or H-8), $4.24(\mathrm{~d}, J=4.1 \mathrm{~Hz}, 1 \mathrm{H}, \mathrm{H}-2$ or H-8), 4.03-3.97 (m, 1H, H-5), 3.93 (ddd, $J=$ 9.5, 3.9, and $3.9 \mathrm{~Hz}, 1 \mathrm{H}, \mathrm{H}-3$ ), 3.86 (ddd, $J=7.3,7.3$, and $4.0 \mathrm{~Hz}, 1 \mathrm{H}, \mathrm{H}-7), 3.47$ (s, 3H, OMe), 3.46 (s, $3 \mathrm{H}, \mathrm{OMe}), 3.40$ (s, 3H, $\mathrm{OCH}_{2} \mathrm{OMe}$ ), 3.39 (s, 3H, $\mathrm{OCH}_{2} \mathrm{OMe}$ ), and 1.75-1.61 (m, 4H, H-4 and H-6).

${ }^{13} \mathrm{C}$ NMR $\left(125 \mathrm{MHz}, \mathrm{CDCl}_{3}\right): \delta=171.5,96.8,81.8,78.7,76.5,67.4,59.4,58.7,56.6,56.5,52.3,39.2$, and 38.0.

HR ESI-MS: Calcd for $\mathrm{C}_{17} \mathrm{H}_{32} \mathrm{O}_{11} \mathrm{Na}(\mathrm{M}+\mathrm{Na})^{+}: 435.1842$ Found: 435.1829.

TLC: $R_{\mathrm{f}}=0.8 ; 100 \%$ ethyl acetate. 
$(3 R, 4 R, 8 R, 9 R)-4,8-D i m e t h o x y-3,9-b i s(m e t h o x y m e t h o x y)$ undeca-1,10-dien-6-ol (2)

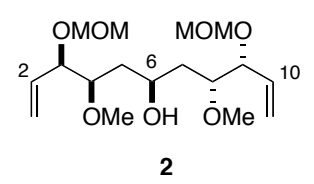

${ }^{1} \mathbf{H}$ NMR $\left(500 \mathrm{MHz}, \mathrm{CDCl}_{3}\right): \delta$ = 5.82-5.71 (m, 2H, H-2 and H-10), 5.35-5.26 (m, 4H, H-1 and H-11) $4.71\left(\mathrm{~d}, J=6.4 \mathrm{~Hz}, 1 \mathrm{H}, \mathrm{OCH}_{\mathrm{a} 1} \mathrm{HOMe}\right), 4.69\left(\mathrm{~d}, J=6.4 \mathrm{~Hz}, 1 \mathrm{H}, \mathrm{OCH}_{\mathrm{a} 2} \mathrm{HOMe}\right), 4.60(\mathrm{~d}, J=6.4 \mathrm{~Hz}, 1 \mathrm{H}$, $\left.\mathrm{OCH}_{\mathrm{b} 1} \mathrm{OMe}\right), 4.58\left(\mathrm{~d}, J=6.4 \mathrm{~Hz}, 1 \mathrm{H}, \mathrm{OCH} H_{\mathrm{b} 2} \mathrm{OMe}\right), 4.20(\mathrm{ddd}, J=7.2,5.0$, and $1.0 \mathrm{~Hz}, 1 \mathrm{H}, \mathrm{H}-3$ or H9), 4.13 (ddd, $J=7.2,5.0$, and $1.0 \mathrm{~Hz}, 1 \mathrm{H}, \mathrm{H}-3$ or H-9), 4.02 (dddd, $J=9.9,9.9,2.8$, and $2.8 \mathrm{~Hz}, 1 \mathrm{H}, \mathrm{H}-$ 6), 3.60-3.47 (m, 2H, H-4 and H-8), 3.53 (s, 3H, OMe), 3.52 (s, 3H, OMe), 3.39 (s, 3H, $\mathrm{OCH}_{2} \mathrm{OMe}$ ), 3.38 (s, 3H, $\mathrm{OCH}_{2} \mathrm{OMe}$ ), and 1.75-1.48 (m, 4H, H-5 and $\left.\mathrm{H}-7\right)$.

${ }^{13} \mathrm{C}$ NMR $\left(125 \mathrm{MHz}, \mathrm{CDCl}_{3}\right): \delta=134.9,134.2,119.1,118.7,94.4,94.3,83.6,79.8,78.6,77.6,67.8$, $59.6,58.7,55.8,55.7,39.9$, and 37.8 .

HR ESI-MS: Calcd for $\mathrm{C}_{17} \mathrm{H}_{32} \mathrm{O}_{7} \mathrm{Na}(\mathrm{M}+\mathrm{Na})^{+}: 371.2046$ Found: 371.2020.

TLC: $\mathrm{R}_{\mathrm{f}}=0.6 ; 50 \%$ ethyl acetate: $50 \%$ hexanes. 
$(8 S, 7 R, 5 S)-5-[(2 S, 3 R)-1,3-D i m e t h o x y-2-(m e t h o x y m e t h o x y)-1-0 x o b u t y l]-7-m e t h o x y-8-$

(methoxymethoxy)tetrahydropyran-1-one (3-cis)

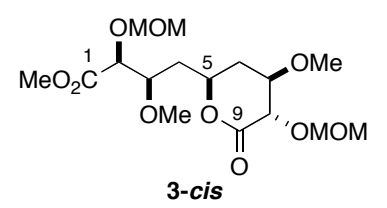

${ }^{1} \mathbf{H}$ NMR $\left(500 \mathrm{MHz}, \mathrm{CDCl}_{3}\right): \delta=5.02\left[\mathrm{~d}, J=6.8 \mathrm{~Hz}, 1 \mathrm{H} \mathrm{C}(8)-\mathrm{OC} H_{\mathrm{a} 1} \mathrm{HOMe}\right], 4.80[\mathrm{~d}, J=6.8 \mathrm{~Hz}, 1 \mathrm{H}$ $\left.\mathrm{C}(8)-\mathrm{OCH}_{\mathrm{a} 2} \mathrm{HOMe}\right], 4.73\left[\mathrm{~d}, J=8.0 \mathrm{~Hz}, 1 \mathrm{H}, \mathrm{C}(2)-\mathrm{OCH} H_{\mathrm{b} 1} \mathrm{OMe}\right], 4.72[\mathrm{~d}, J=7.0 \mathrm{~Hz}, 1 \mathrm{H}, \mathrm{C}(2)-$

OCH $H_{\mathrm{b} 2} \mathrm{OMe}$ ) 4.47 (m, 1H, H-5), 4.28 (d, $\left.J=3.6 \mathrm{~Hz}, 1 \mathrm{H}, \mathrm{H}-2\right), 4.16$ (d, $J=8.0 \mathrm{~Hz}, 1 \mathrm{H}, \mathrm{H}-8$ ), 3.91 (ddd, $J=6.6,6.6$, and $3.3 \mathrm{~Hz}, 1 \mathrm{H}, \mathrm{H}-3), 3.78\left(\mathrm{~s}, 3 \mathrm{H}, \mathrm{CO}_{2} \mathrm{Me}\right), 3.65(\mathrm{ddd}, J=10.2,8.0$, and $5.0 \mathrm{~Hz}, 1 \mathrm{H}, \mathrm{H}-7)$, 3.47 (s, 3H, OMe), 3.46 (s, 3H, OMe), 3.40 (s, 3H, OCH $\mathrm{O}_{2} \mathrm{OMe}$ ), $3.38\left(\mathrm{~s}, 3 \mathrm{H}, \mathrm{OCH}_{2} \mathrm{OMe}\right.$ ), 2.41 (ddd, $J=$ 14.0, 4.9, and $2.9 \mathrm{~Hz}, 1 \mathrm{H}, \mathrm{H}-6 \mathrm{eq}), 1.72$ (ddd, $J=14.1,11.0$, and 10.0 Hz, H-6ax), and 2.14-1.93 (m, 2H, H-4).

${ }^{13} \mathbf{C ~ N M R}\left(75 \mathrm{MHz}, \mathrm{CDCl}_{3}\right): \delta=171.0,170.7,97.1,96.9,77.8,76.3,75.2,73.7,66.1,58.3,57.6,56.6$, 56.4 , and $52.4,35.9$, and 34.3 .

HR ESI-MS: Calcd for $\mathrm{C}_{16} \mathrm{H}_{28} \mathrm{O}_{10} \mathrm{Na}(\mathrm{M}+\mathrm{Na})^{+}: 403.1580$ Found: 403.1585 .

TLC: $R_{\mathrm{f}}=0.25 ; 50 \%$ ethyl acetate: $50 \%$ hexanes. 


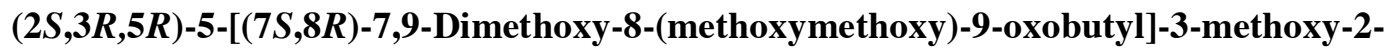

(methoxymethoxy)tetrahydropyran-1-one (3-trans)

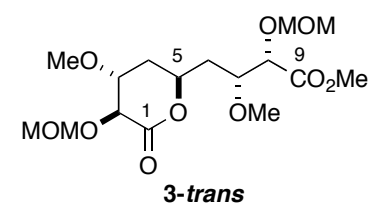

${ }^{1} \mathbf{H}$ NMR $\left(300 \mathrm{MHz}, \mathrm{CDCl}_{3}\right): \delta=4.94\left[\mathrm{~d}, J=6.9 \mathrm{~Hz}, 1 \mathrm{H} \mathrm{C}(2)-\mathrm{OCH}_{\mathrm{a} 1} \mathrm{HOMe}\right], 4.77[\mathrm{~d}, J=6.9 \mathrm{~Hz}, 1 \mathrm{H}$ $\left.\mathrm{C}(2)-\mathrm{OCH} H_{\mathrm{b} 1} \mathrm{OMe}\right], 4.74\left[\mathrm{~m}, 1 \mathrm{H}, \mathrm{H}-5\right.$, (gcosy analysis)], $4.72\left[\mathrm{~d}, J=6.9 \mathrm{~Hz}, 1 \mathrm{H}, \mathrm{C}(8)-\mathrm{OCH}_{\mathrm{a} 2} \mathrm{HOMe}\right]$, $4.70\left[\mathrm{~d}, J=6.9 \mathrm{~Hz}, 1 \mathrm{H}, \mathrm{C}(8)-\mathrm{OCH} H_{\mathrm{b} 2} \mathrm{OMe}\right], 4.39$ (d, $\left.J=6.4 \mathrm{~Hz}, 1 \mathrm{H}, \mathrm{H}-2\right), 4.23(\mathrm{~d}, J=4.2 \mathrm{~Hz}, 1 \mathrm{H}, \mathrm{H}-8)$, 3.95 (ddd, $J=8.8,6.5$, and $4.1 \mathrm{~Hz}, 1 \mathrm{H}, \mathrm{H}-7), 3.78\left(\mathrm{~s}, 3 \mathrm{H}, \mathrm{CO}_{2} \mathrm{Me}\right), 3.65$ (ddd, $J=6.3,6.3$, and $2.5 \mathrm{~Hz}$, 1H, H-3), 3.47 (s, 3H, OMe), 3.45 (s, 3H, OMe), 3.42 (s, 3H, $\left.\mathrm{OCH}_{2} \mathrm{OMe}\right), 3.40$ (s, 3H, $\left.\mathrm{OCH}_{2} \mathrm{OMe}\right)$, and 2.10-1.62 (m, 4H, H-4 and H-6).

${ }^{13} \mathrm{C} \mathrm{NMR}\left(75 \mathrm{MHz}, \mathrm{CDCl}_{3}\right): \delta=171.0,170.7,96.7,96.2,76.2,73.9,71.9,59.5,57.4,56.2,52.2,37.2$, and 34.8.

HR ESI-MS: Calcd for $\mathrm{C}_{16} \mathrm{H}_{28} \mathrm{O}_{10} \mathrm{Na}(\mathrm{M}+\mathrm{Na})^{+}: 403.1580$ Found: 403.1598.

TLC: $\mathrm{R}_{\mathrm{f}}=0.3 ; 50 \%$ ethyl acetate: $50 \%$ hexanes. 
(2S,3R,5R)- and (2S,3R,5S)-Methyl 5-Hydroxy-3-methoxy-2-(methoxymethoxy)dodecanoate (4R and $4 S$ )

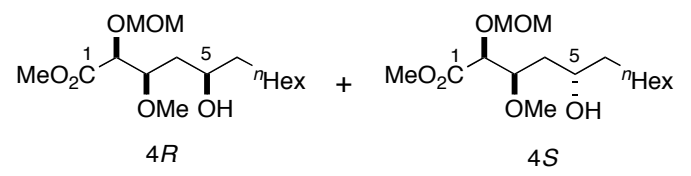

${ }^{1} \mathbf{H}$ NMR $\left(300 \mathrm{MHz}, \mathrm{CDCl}_{3}\right): \delta=4.73\left(\mathrm{~s}, 2 \mathrm{H}, \mathrm{OCH}_{2} \mathrm{OCH}_{3}\right), 4.73\left(\mathrm{~s}, 2 \mathrm{H}, \mathrm{OCH}_{2} \mathrm{OCH}_{3}\right), 4.28(\mathrm{~d}, J=4.0$, 1H, H-2), 4.26 (d, $J=4.0,1 \mathrm{H}, \mathrm{H}-2$ ), 3.90 (ddd, $J=8.4,4.2$, and 4.2 Hz, 1H, H-3), 3.89 (ddd, $J=8.4,3.9$, and $3.9 \mathrm{~Hz}, 1 \mathrm{H}, \mathrm{H}-3$ ), 3.83 (nfom, 1H, H-5), 3.83 (nfom, 1H, H-5), 3.79 (s, 3H, $\mathrm{CO}_{2} \mathrm{Me}$ ), 3.78 (s, 3H, $\mathrm{CO}_{2} \mathrm{Me}$ ), 3.47 (s, 3H, OMe), 3.46 (s, 3H, OMe), 3.41 (s, 3H, $\mathrm{OCH}_{2} \mathrm{OMe}$ ), 3.41 (s, 3H, $\mathrm{OCH}_{2} \mathrm{OMe}$ ), 1.75 (ddd, $J=14.4,8.4$, and $2.7 \mathrm{~Hz}, 1 \mathrm{H}, \mathrm{H}_{\mathrm{a}}-4$ of $4 S$ ), 1.71 (nfom, 2)H, $\mathrm{H}-4$ of $4 R$ ), 1.62 (ddd, $J=14.4,9.6$, and $3.9 \mathrm{~Hz}, 1 \mathrm{H}, \mathrm{H}_{\mathrm{b}}-4$ of $\left.4 \mathrm{~S}\right), 1.36-1.23(\mathrm{~m}, 12 \mathrm{H}), 1.36-1.23(\mathrm{~m}, 12 \mathrm{H}), 0.88\left(\mathrm{t}, J=6.4 \mathrm{~Hz}, 3 \mathrm{H}, \mathrm{CH}_{2} \mathrm{CH}_{3}\right)$, and $0.88\left(\mathrm{t}, J=6.4 \mathrm{~Hz}, 3 \mathrm{H}, \mathrm{CH}_{2} \mathrm{CH}_{3}\right)$.

${ }^{13} \mathrm{C}$ NMR (75 MHz, $\left.\mathrm{CDCl}_{3}\right)$ : Complex - lactonization in $\mathrm{CDCl}_{3}$ occurred during data collection.

HR ESI-MS: Calcd for $\mathrm{C}_{16} \mathrm{H}_{32} \mathrm{O}_{6} \mathrm{Na}(\mathrm{M}+\mathrm{Na})^{+}: 343.2097$ Found: 343.2079.

TLC: $\mathrm{R}_{\mathrm{f}}=0.3 ; 30 \%$ ethyl acetate: $70 \%$ hexanes. 
$(2 S, 3 R, 5 R)$ - and $(2 S, 3 R, 5 S)$-Methyl 5-(Benzyloxy)-3-methoxy-2-(methoxymethoxy)dodecanoate (Bn$4 R$ and Bn-4S, the benzyl ether precursors to $4 R$ and $4 S$ )

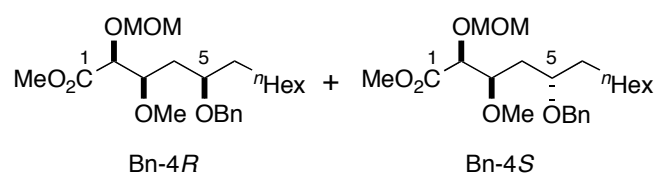

${ }^{1} \mathbf{H}$ NMR $\left(500 \mathrm{MHz}, \mathrm{CDCl}_{3}\right): \delta=7.38-7.27(\mathrm{~m}, 5 \mathrm{H}, \mathrm{Ar}-\mathrm{H})$, 7.38-7.27 (m, 5H, Ar-H), $4.72(\mathrm{~d}, J=6.9 \mathrm{~Hz}$, $\left.1 \mathrm{H}, \mathrm{OC} H_{\mathrm{a} 1} \mathrm{HOMe}\right), 4.71\left(\mathrm{~d}, J=6.9 \mathrm{~Hz}, 1 \mathrm{H}, \mathrm{OCH}_{\mathrm{a} 2} \mathrm{HOMe}\right), 4.69\left(\mathrm{~d}, J=6.9 \mathrm{~Hz}, 1 \mathrm{H}, \mathrm{OCH}_{\mathrm{b} 1} \mathrm{OMe}\right), 4.68$ $\left(\mathrm{d}, J=6.9 \mathrm{~Hz}, 1 \mathrm{H}, \mathrm{OCH}_{\mathrm{b} 2} \mathrm{OMe}\right), 4.61\left(\mathrm{~d}, J=11.4 \mathrm{~Hz}, 1 \mathrm{H}, \mathrm{PhCH}_{\mathrm{a} 1} \mathrm{HO}\right), 4.55(\mathrm{~d}, J=11.4 \mathrm{~Hz}, 1 \mathrm{H}$, $\left.\mathrm{PhCH} H_{\mathrm{b} 1} \mathrm{O}\right), 4.48\left(\mathrm{~d}, J=11.4 \mathrm{~Hz}, 1 \mathrm{H}, \mathrm{PhCH}_{\mathrm{a} 2} \mathrm{HO}\right), 4.43\left(\mathrm{~d}, J=11.4 \mathrm{~Hz}, 1 \mathrm{H}, \mathrm{PhCH} H_{\mathrm{b} 2} \mathrm{O}\right), 4.19(\mathrm{~d}, J=4.0$ $\mathrm{Hz}, 1 \mathrm{H}, \mathrm{H}-2), 4.15(\mathrm{~d}, J=3.5 \mathrm{~Hz}, 1 \mathrm{H}, \mathrm{H}-2), 3.86(\mathrm{ddd}, J=9.4,4.0$, and $4.0 \mathrm{~Hz}, 1 \mathrm{H}, \mathrm{H}-3), 3.83$ (ddd, $J=$ 7.0, 4.0, and 4.0 Hz, 1H, H-3), 3.77 (s, 3H, OMe), 3.77 (s, 3H, OMe), 3.62 (dddd, $J=9.5,6.5,4.5$, and $3.0 \mathrm{~Hz}, 1 \mathrm{H}, \mathrm{H}-5$ ), 3.49 (nfom, 1H, H-5), 3.39 (s, 3H, OMe), 3.39 (s, 3H, OMe), 3.36 (s, 3H, OCH${ }_{2} \mathrm{OMe}$ ), $3.32\left(\mathrm{~s}, 3 \mathrm{H}, \mathrm{OCH}_{2} \mathrm{OMe}\right), 1.96\left(\mathrm{ddd}, J=14.5,7.5\right.$, and $6.5 \mathrm{~Hz}, 1 \mathrm{H}, \mathrm{H}_{\mathrm{a}}-4$ for $\left.\mathrm{Bn}-4 R\right) 1.80(\mathrm{ddd}, J=14.5$, 7.0, and 5.0 Hz, 1H, $\mathrm{H}_{\mathrm{b}}-4$ for $\mathrm{Bn}-4 R$ ), 1.75 (ddd, $J=14.5,9.5$, and $3.0 \mathrm{~Hz}, 1 \mathrm{H}, \mathrm{H}_{\mathrm{a}}-4$ for $\mathrm{Bn}-4 S$ ) 1.70 (ddd, $J=14.5,10.0$, and $3.5 \mathrm{~Hz}, 1 \mathrm{H}, \mathrm{H}_{\mathrm{b}}-4$ for $\left.\mathrm{Bn}-4 S\right), 1.7-1.5(\mathrm{~m}, 2 \mathrm{H}, \mathrm{H}-6), 1.7-1.5$ (m, 2H, H-6), 1.43-1.20 (m, $12 \mathrm{H}), 1.43-1.20(\mathrm{~m}, 12 \mathrm{H}), 0.89\left(\mathrm{t}, J=5.5 \mathrm{~Hz}, 6 \mathrm{H}, \mathrm{CH}_{2} \mathrm{CH}_{3}\right)$, and $0.89\left(\mathrm{t}, J=5.5 \mathrm{~Hz}, 6 \mathrm{H}, \mathrm{CH}_{2} \mathrm{CH}_{3}\right)$.

${ }^{13} \mathrm{C} \mathrm{NMR}\left(75 \mathrm{MHz}, \mathrm{CDCl}_{3}\right): \delta=171.3,171.3,128.3,127.9,127.8,127.8,127.5,127.5,96.7,96.6,78.6$, $78.2,77.5,77.1,76.7,75.4,70.7,70.6,58.9,58.2,56.4,56.3,52.0,51.9,36.3,34.4,33.9,33.8,31.9,31.8$, $29.9,29.8,29.3,29.1,25.1,24.9,22.7$, and 14.2.

HR ESI-MS: Calcd for $\mathrm{C}_{23} \mathrm{H}_{38} \mathrm{O}_{6} \mathrm{Na}(\mathrm{M}+\mathrm{Na})^{+}: 433.2566$ Found: 433.2568 .

TLC: $\mathrm{R}_{\mathrm{f}}=0.4 ; 30 \%$ ethyl acetate: $70 \%$ hexanes. 


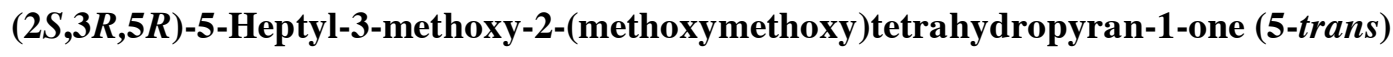

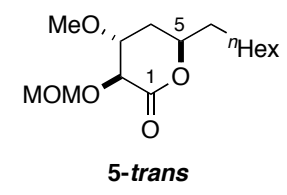

${ }^{1} \mathbf{H}$ NMR $\left(500 \mathrm{MHz}, \mathrm{CDCl}_{3}\right): \delta=4.93\left(\mathrm{~d}, J=6.4 \mathrm{~Hz}, 1 \mathrm{H}, \mathrm{OCH}_{\mathrm{a}} \mathrm{HOMe}\right), 4.77(\mathrm{~d}, J=6.4 \mathrm{~Hz}, 1 \mathrm{H}$, OCH $\left.H_{\mathrm{b}} \mathrm{OMe}\right), 4.37$ (d, $\left.J=6.5 \mathrm{~Hz}, 1 \mathrm{H}, \mathrm{H}-2\right), 4.47$ (m, 1H, H-5), 3.64 (ddd, $J=6.5,6.5$, and $2.0 \mathrm{~Hz}, 1 \mathrm{H}$, H-3), 3.46 (s, 3H, OMe), 3.46 (s, 3H, $\mathrm{OCH}_{2} M e$ ), 2.01 (ddd, $J=15.1,2.4$, and 2.4 Hz, 1H, H-4eq), 1.91 (ddd, $J=15.0,11.2$, and $6.7 \mathrm{~Hz}, 1 \mathrm{H}, \mathrm{H}-4 \mathrm{ax}), 1.70$ (m, 1H, H-6), 1.55 (m, 1H, H-6), 1.50-1.20 (m, 10H), and $0.89\left(\mathrm{t}, \mathrm{J}=6.2 \mathrm{~Hz}, 3 \mathrm{H}, \mathrm{CH}_{2} \mathrm{CH}_{3}\right)$.

${ }^{13} \mathrm{C}$ NMR (125 MHz, $\left.\mathrm{CDCl}_{3}\right): \delta=170.7,97.0,77.9,75.4,74.0,57.5,56.3,35.8,34.6,31.9,29.4,29.3$, 25.0, 22.8, and 14.2.

HR ESI-MS: Calcd for $\mathrm{C}_{15} \mathrm{H}_{28} \mathrm{O}_{5} \mathrm{Na}(\mathrm{M}+\mathrm{Na})^{+}: 311.1834$ Found: 311.1803.

TLC: $\mathrm{R}_{\mathrm{f}}=0.2 ; 30 \%$ ethyl acetate: $70 \%$ hexanes. 


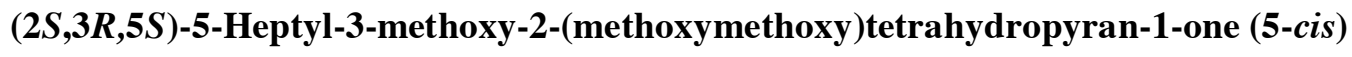

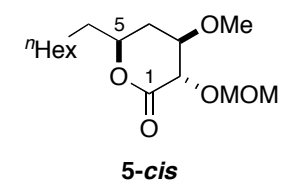

${ }^{1} \mathbf{H}$ NMR $\left(500 \mathrm{MHz}, \mathrm{CDCl}_{3}\right): \delta=5.04\left(\mathrm{~d}, J=6.2 \mathrm{~Hz}, 1 \mathrm{H}, \mathrm{OCH}_{\mathrm{a}} \mathrm{HOMe}\right), 4.80(\mathrm{~d}, J=6.2 \mathrm{~Hz}, 1 \mathrm{H}$, $\mathrm{OCH}_{\mathrm{b}} \mathrm{OMe}$ ), 4.28 (m, 1H, H-5), $4.13(\mathrm{~d}, J=8.5 \mathrm{~Hz}, 1 \mathrm{H}, \mathrm{H}-2), 3.64$ (ddd, $J=10.6,8.3$, and $5.0 \mathrm{~Hz}, 1 \mathrm{H}$, H-3), 3.48 (s, 3H, OMe), 3.47 (s, 3H, OMe), 2.34 (ddd, $J=13.5,4.8$, and $2.7 \mathrm{~Hz}, 1 \mathrm{H}, \mathrm{H}-4 \mathrm{eq}), 1.80-1.60$ (nfom, 1H, H-4ax), 1.80-1.60 (m, 2H, H-6), 1.50-1.20 (m, 10H), and 0.90 (t, $J=7.0 \mathrm{~Hz}, 3 \mathrm{H}, \mathrm{CH}_{2} \mathrm{CH}_{3}$ ).

${ }^{13} \mathrm{C}$ NMR (125 MHz, $\left.\mathrm{CDCl}_{3}\right): \delta=170.9,96.2,77.7,75.4,74.0,57.5,56.2,35.1,34.6,31.9,29.4,29.3$, 25.1, 22.8, and 14.3.

HR ESI-MS: Calcd for $\mathrm{C}_{15} \mathrm{H}_{28} \mathrm{O}_{5} \mathrm{Na}(\mathrm{M}+\mathrm{Na})^{+}: 311.1834$ Found: 311.1803.

TLC: $\mathbf{R}_{\mathrm{f}}=0.2 ; 30 \%$ ethyl acetate: $70 \%$ hexanes. 
$(3 R, 4 R, 6 R)-4-M e t h o x y-3-(m e t h o x y m e t h o x y) t r i d e c-1-e n-6-o l(8 R)$

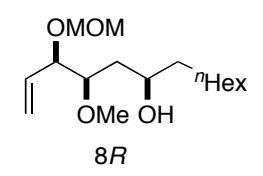

${ }^{1} \mathbf{H}$ NMR [from a mixture enriched in this 4,6-syn diastereomer $(\mathbf{8 R}) ; 500 \mathrm{MHz}, \mathrm{CDCl}_{3}$ ]: $\mathbf{S y n}: \delta=5.76$ (ddd, $1 \mathrm{H}, J=17.1,10.0$, and $7.2 \mathrm{~Hz}, 1 \mathrm{H}, \mathrm{H}-2), 5.35-5.29(\mathrm{~m}, 2 \mathrm{H}, \mathrm{H}-1), 4.70(\mathrm{~d}, J=6.7 \mathrm{~Hz}, 1 \mathrm{H}$, $\mathrm{OCH}_{\mathrm{a}} \mathrm{HOMe}$ ), 4.59 (d, $J=6.7 \mathrm{~Hz}, 1 \mathrm{H}, \mathrm{OCH} H_{\mathrm{b}} \mathrm{OMe}$ ), 4.20 (br dd, $J=7$ and $\left.7 \mathrm{~Hz}, \mathrm{H}-3\right), 3.79$ (br m, 1H, H-4), 3.6-3.5 (m, 1H, H-6), 3.52 (s, 3H, $\mathrm{OCH}_{2} \mathrm{OMe}$ ), 3.39 (s, 3H, OMe), 1.71 (ddd, $J=14.8,3.8$, and 2.6 $\left.\mathrm{Hz}, 1 \mathrm{H}, \mathrm{H}_{\mathrm{a}}-5\right), 1.53\left(\mathrm{ddd}, J=14.7,9.6\right.$, and $\left.9.6 \mathrm{~Hz}, 1 \mathrm{H}, \mathrm{H}_{\mathrm{b}}-5\right), 1.36-1.23(\mathrm{~m}, 12 \mathrm{H})$, and $0.89(\mathrm{t}, J=8.0$ $\left.\mathrm{Hz}, 3 \mathrm{H}, \mathrm{CH}_{2} \mathrm{CH}_{3}\right)$.

${ }^{13} \mathbf{C}$ NMR $\left(75 \mathrm{MHz}, \mathrm{CDCl}_{3}\right): \delta=134.7,119.0,94.3,80.9,78.5,68.9,59.2,55.7,38.3,37.6,31.9,29.8$, $29.5,25.9,22.8$, and 14.3.

HR ESI-MS: Calcd for $\mathrm{C}_{16} \mathrm{H}_{32} \mathrm{O}_{4} \mathrm{Na}(\mathrm{M}+\mathrm{Na})^{+}: 311.2198$ Found: 311.2173.

TLC: $\mathrm{R}_{\mathrm{f}}=0.4 ; 30 \%$ ethyl acetate: $70 \%$ hexanes. 
$(3 R, 4 R, 6 S)-4-M e t h o x y-3-(m e t h o x y m e t h o x y) t r i d e c-1-e n-6-o l ~(8 S)$

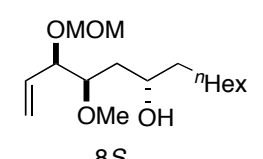

$8 S$

${ }^{1} \mathbf{H}$ NMR [from a mixture enriched in the 4,6-anti diastereomer (8S); $500 \mathrm{MHz}, \mathrm{CDCl}_{3}$ ]: Anti: $\delta=5.75$

(ddd, $1 \mathrm{H}, J=17.5,10.0$, and $7.3 \mathrm{~Hz}, 1 \mathrm{H}, \mathrm{H}-2), 5.35-5.29(\mathrm{~m}, 2 \mathrm{H}, \mathrm{H}-1), 4.71(\mathrm{~d}, J=6.6 \mathrm{~Hz}, 1 \mathrm{H}$,

$\left.\mathrm{OCH}_{\mathrm{a}} \mathrm{HOMe}\right), 4.60\left(\mathrm{~d}, J=6.7 \mathrm{~Hz}, 1 \mathrm{H}, \mathrm{OCH} H_{\mathrm{b}} \mathrm{OMe}\right), 4.20$ (br dd, $J=7$ and $\left.7 \mathrm{~Hz}, \mathrm{H}-3\right), 3.79$ (br m, 1H,

H-4), 3.6-3.5 (m, 1H, H-6), 3.51 (s, 3H, $\mathrm{OCH}_{2} \mathrm{OMe}$ ), 3.40 (s, 3H, OMe), 1.62 (nfom, 2H, H-5), 1.36-1.23

(m, 12H), and $0.89\left(\mathrm{t}, J=8.0 \mathrm{~Hz}, 3 \mathrm{H}, \mathrm{CH}_{2} \mathrm{CH}_{3}\right)$.

${ }^{13} \mathbf{C}$ NMR $\left(75 \mathrm{MHz}, \mathrm{CDCl}_{3}\right): \delta=134.2,119.1,94.4,83.9,77.7,71.5,58.7,55.8,37.9,37.1,31.9,29.8$, 29.5, 25.6, 22.8, and 14.3.

HR ESI-MS: Calcd for $\mathrm{C}_{16} \mathrm{H}_{32} \mathrm{O}_{4} \mathrm{Na}(\mathrm{M}+\mathrm{Na})^{+}: 311.2198$ Found: 311.2173 .

TLC: $\mathrm{R}_{\mathrm{f}}=0.4 ; 30 \%$ ethyl acetate: $70 \%$ hexanes. 

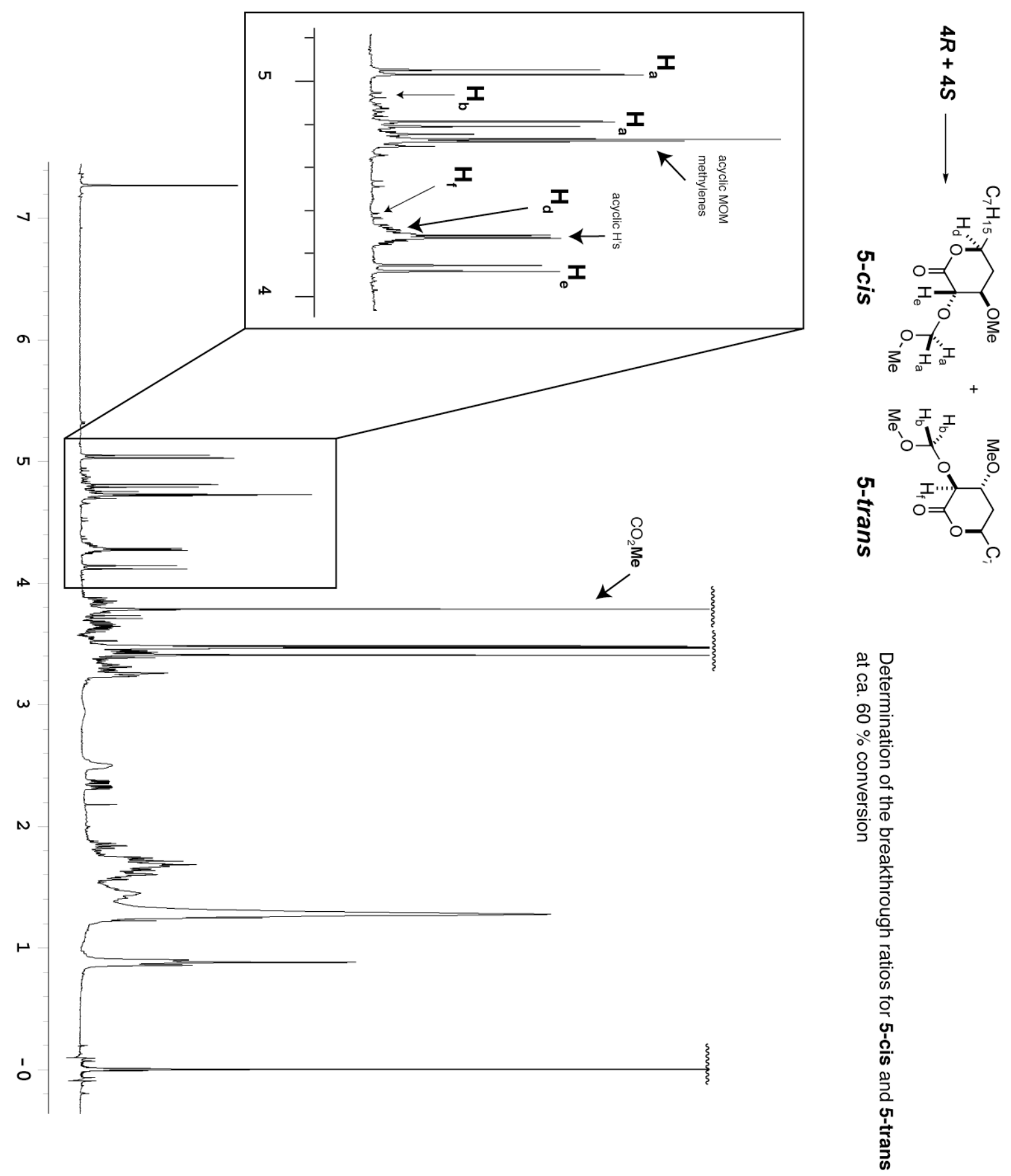


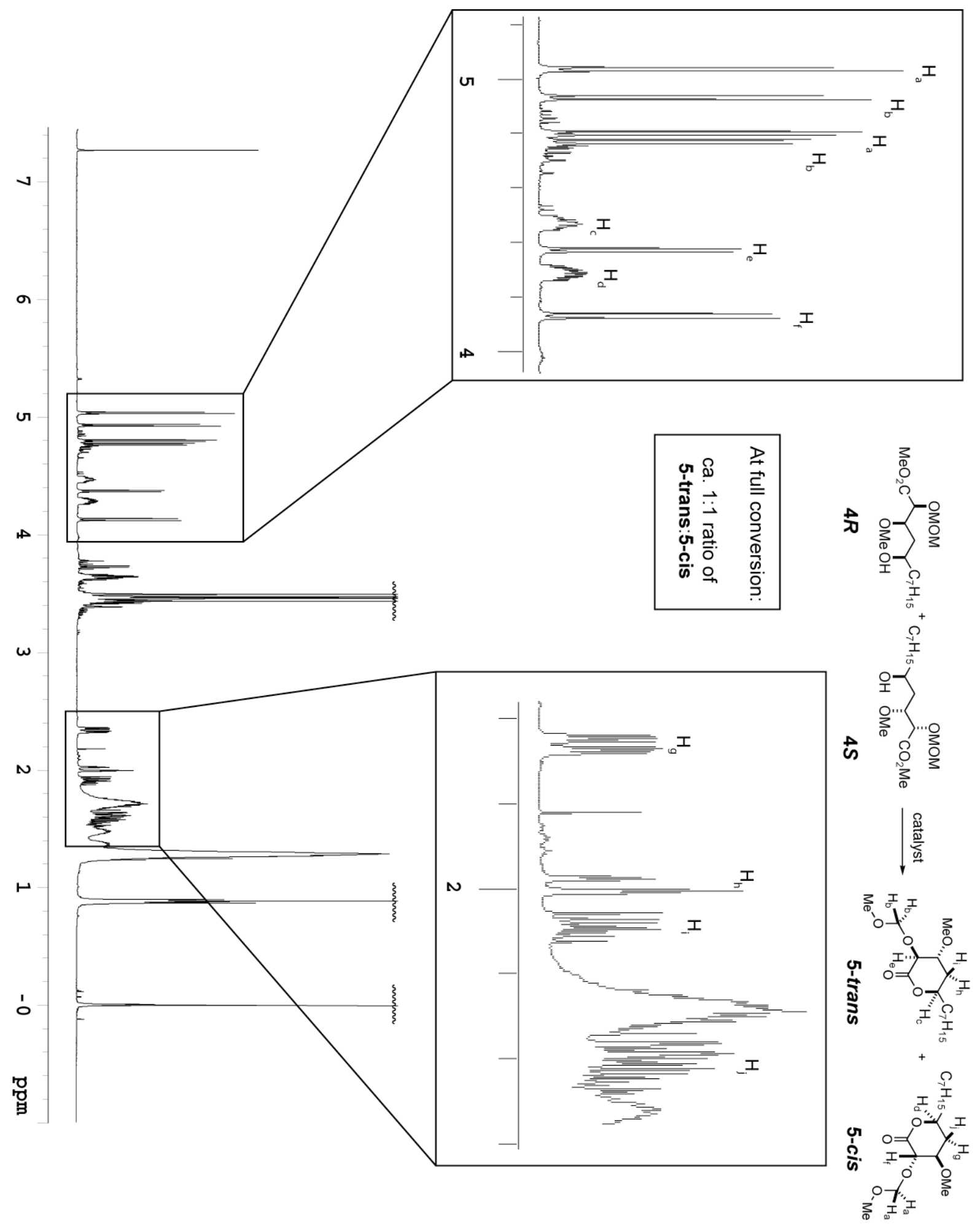




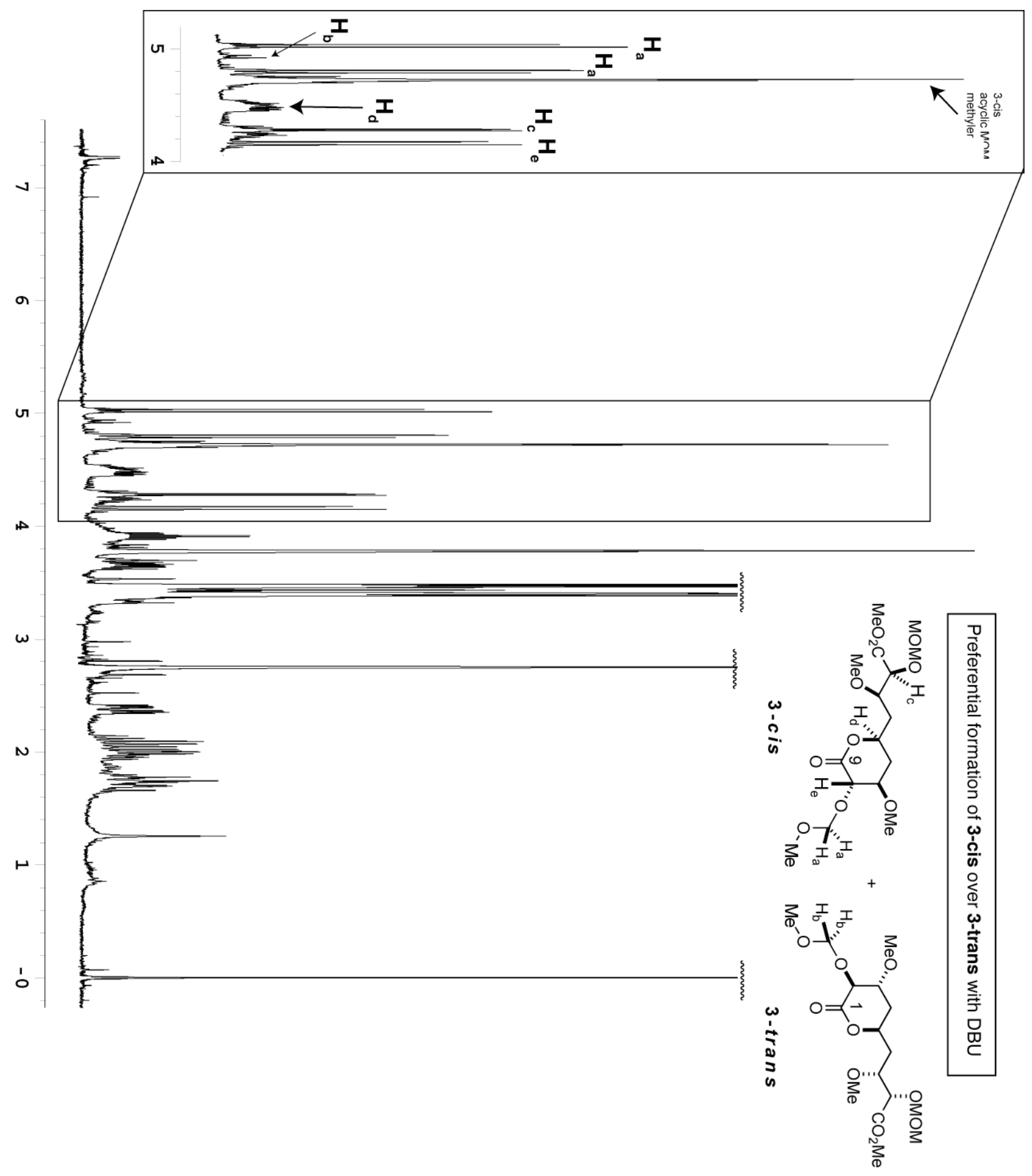




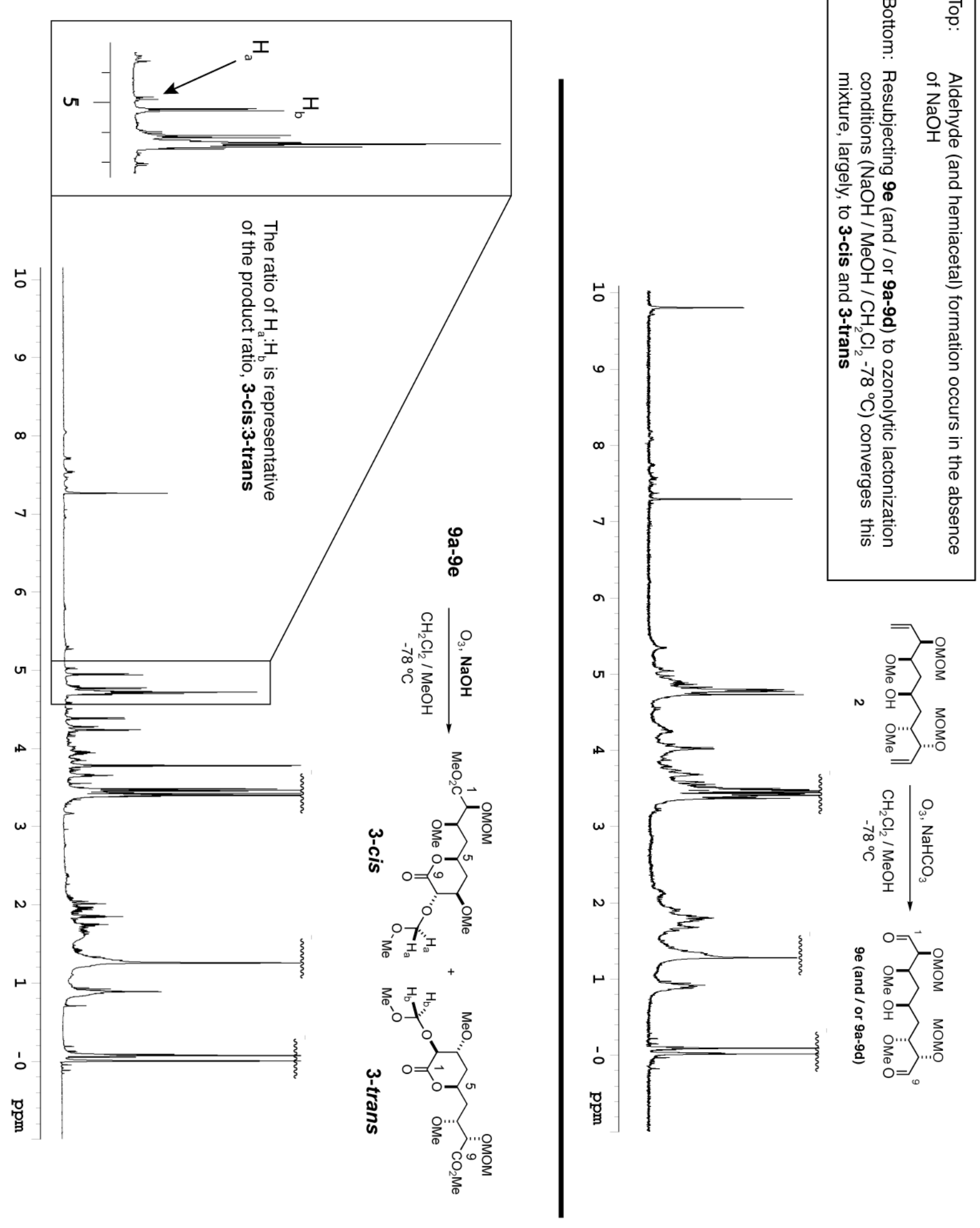

